\title{
Properties of feature detectors for speech: Evidence from the effects of selective adaptation on dichotic listening
}

\author{
JOANNE L. MILLER \\ Brown University, Providence, Rhode Island 02912
}

\begin{abstract}
Two selective adaptation experiments were conducted in order to investigate certain properties of feature detector systems sensitive to the information underlying the voicing distinction (Experiment I) and the place of articulation distinction (Experiment II). The experimental paradigm combined binaural adaptation with a dichotic testing procedure. The stimuli were nonboundary, good exemplars of the respective phonetic categories. In both experiments, there was a systematic shift in performance following adaptation on those trials on which the stimulus in one ear had the adapted feature value and the stimulus in the other ear had the unadapted feature value. On these trials, the adapted feature value was relatively less effective in competing for processing with the unadapted value in the opposing ear (compared to preadaptation performance). Since these results were obtained using nonboundary stimuli, it was argued that (1) adaptation affects the relevant detector along its range of operation or sensitivity, and not simply at the phonetic boundary, and that (2) information regarding the relative output level of the detector, as well as which detector was more strongly excited, must be available at the site of interaction of the two stimuli.
\end{abstract}

At some stage of processing, the perception of speech involves the extraction of phonetic features from the acoustic signal. There is a great deal of evidence that this entails more than a simple mapping of acoustic to phonetic segments, and that a speech-specific processing system is required for the task (see Liberman, Cooper, Shankweiler, \& Studdert-Kennedy, 1967; Studdert-Kennedy, 1974). It has recently been proposed that feature extraction is accomplished by sets of detectors that are specifically tuned to the relevant information underlying feature distinctions, and that yield as their output the equivalent of discrete phonetic feature values (e.g., Abbs \& Sussman, 1971; Eimas \& Corbit, 1973).

The evidence supporting a detector model of feature extraction comes primarily from selective adaptation experiments. Although this technique has an extensive history of use with both visual stimuli (see Sekular, 1974) and nonspeech auditory stimuli (see, for example, Ward, 1973), it has been employed only recently in the investigation of speech processing. Eimas and Corbit (1973) were the first to use this technique with speech, and subsequent research has been patterned after their initial experiments.

This research was supported by Grant NS 00143-01 from the National Institute of Neurological Diseases and Stroke to the author and by Grant HD 05331 from the National Institute of Child Health and Human Development to Dr. Peter D. Eimas. I wish to express my gratitude to Dr. Franklin S. Cooper and Dr. Alvin M. Liberman for their generosity in making available the facilities of the Haskins Laboratories for the preparation of stimulus materials, and would like to thank Dr. Peter D. Eimas for his very helpful comments on an earlier version of this manuscript.
The typical adaptation procedure, as developed by Eimas and Corbit, proceeds as follows. The listener is asked to identify a series of synthetic speech stimuli varying along an acoustic continuum that underlies a specific linguistic distinction, typically voicing or place of articulation. As expected from the extensive literature on the identification of such continua (see Liberman et al., 1967), the stimuli are readily assigned to discrete phonetic categories. After identification, the listener is repeatedly exposed to an exemplar of one of the categories, and is then presented the stimulus series for identification again. After adaptation, there is a shift in the phonetic boundary toward the adapted category, such that boundary stimuli that were previously identified as belonging to the adapted class are now identified as having the unadapted feature value.

Such boundary shifts have been found for both the voicing (Cooper, 1974b; Eimas, Cooper, \& Corbit, 1973; Eimas \& Corbit, 1973) and the place of articulation dimension (Ades, 1974a; Cooper, 1974a; Cooper \& Blumstein, 1974). By systematically varying the relation between the adapting and test stimuli, it has been shown that adaptation is feature and not phoneme specific (Cooper \& Blumstein, 1974; Eimas $\&$ Corbit, 1973), and that whether the adapting stimulus is perceived as speech is a critical factor (Diehl, 1975; Eimas et al., 1973). In addition, it has been demonstrated that, although the degree of acoustic similarity between the adapting and test stimuli largely determines the degree of adaptation (Tartter \& Eimas, in press), phonetically identical, yet acoustically quite dissimilar, adapting and test stimuli can yield an effect (Ades, 1974b; Diehl, 1975), implicating 
both auditory and phonetic levels of processing. Furthermore, there is evidence to indicate that the effect is perceptual in nature and not merely a reflection of shifts in response criteria (Sawusch \& Pisoni. Note 1; Sawusch, Pisoni, \& Cutting, Note 2).

In order to account for their original adaptation results with stimuli varying along the voice onset time (VOT) continuum (cf. Lisker \& Abramson, 1964), Eimas and Corbit (1973) developed a model of linguistic feature detectors. According to this model. there exist two VOT detectors that have overlapping ranges of sensitivity and that respond maximally to different values of VOT. The detector that is excited more by a given VOT value determines the voicing value assigned to that stimulus, and the phonetic boundary occurs at the point at which the two detectors are equally responsive. Adaptation with an exemplar of one category reduces the output of the corresponding detector along its entire range of sensitivity. This effect causes a shift in the point of equilibrium toward the adapted category, and consequently a shift in the phonetic boundary in that direction. Cooper (1974a) has developed a similar model, involving three linearly arranged detectors, to account for adaptation effects on the place of articulation dimension.

A model of a two-channel feature detector system, with the consequences of adaptation, is displayed in Figure 1. This model, adapted after Eimas and Corbit (1973) and Cooper (1974a), is intended to be a schematic representation of a detector mechanism operating at either an auditory or a phonetic level of analysis. Although the form of the detector system would be the same at both levels, the relevant input dimensions would be quite different. At an auditory level of analysis, the detectors would respond to information coded in the initial auditory transform of the acoustic signal, while at a phonetic level of analysis, the input dimension would presumably be weighted combinations of the outputs of lower order

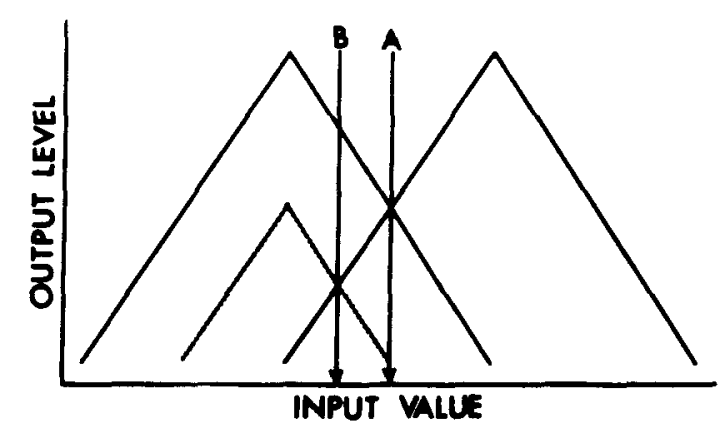

Figure 1. Model of a two-channel feature delector syutem. Output level is given as a function of input value prior to adaptation (solld linea) and following adaptation (dotted lines) with a atimula from one phonetic category. Une A indilicates the location of the preadaptation bonndary, and line $B$ indicates the location of the poutedaptation boundary. auditory detectors. The present set of experiments was aimed at investigating certain aspects of the functioning of a detector system operating at either level. Since no attempt was made to determine the level of operation, the term "feature" will be used throughout the remainder of the paper to refer to information that is either auditory or phonetic in nature. ${ }^{1}$

The model predicts that identification, or more specifically, the assignment of a feature value, is simply a function of which of the opposing channels of analysis is more strongly excited. Accordingly, although the location of the category boundary shifts after adaptation, identification within a given category should remain quite good. And indeed, the boundary shifts found after adaptation generally have not been accompanied by changes in the slope of the identification function (e.g., Diehl, 1975; Eimas \& Corbit, 1973). Anderson (1975) has found that the time taken to classify stimuli also remains constant following adaptation. Using a reaction time task, she found, as did Pisoni and Tash (1974), a peak in the function at the category boundary before adaptation. After adaptation, the peak in reaction time shifted along with the shift in the phonetic boundary, but there was not an overall increase in reaction time for stimuli within the adapted category, nor for that matter was there an increment in reaction time for the actual adapting stimulus.

These results suggest that adaptation does not impair the ability to identify stimuli, but rather that it alters the assignment of feature values, and that it does so only for those stimuli near the phonetic boundary. However, as can be seen from Figure 1, the model assumes that repeated exposure to a stimulus causes a decrease in the sensitivity of the detector along its entire operating range, such that the strength of the output signal is weakened by adaptation even at its point of maximum responsivity. Presumably, identification performance remains high for stimuli within the adapted category because the output of the adapted detector, even though diminished, is still unequivocally greater than that of the opposing channel of analysis. Thus, simple identification procedures are not sufficiently sensitive to provide evidence that adaptation does indeed affect the detector along its entire range of operation. It would be of theoretical importance to develop procedures that would indicate less efficient processing of nonboundary, good exemplars of a category following adaptation. The present set of studies attempted to use a dichotic listening paradigm to accomplish this goal.

The dichotic listening technique has proven to be a valuable tool in the investigation of a number of aspects of speech perception. In this procedure, two different signals are presented to the two ears, and the 
listener is asked to identify what he hears. Performance for both ears is depressed relative to monotic performance, and often in the case of speech stimuli there is an ear differential, with the right ear outperforming the left ear. This right-ear advantage (REA) is taken as evidence for the specialization of the left hemisphere for phonetic feature processing (Studdert-Kennedy \& Shankweiler, 1970).

These experiments have also provided evidence that during processing features are separately extracted from the signal, and are later recombined to form phonetic segments, and that during this processing the signals from the two ears interact. StuddertKennedy and Shankweiler (1970) have argued for feature extraction and convergence on the basis of two kinds of error analysis of dichotic listening data. The stimuli in their experiment were syllables with the initial consonants $[\mathrm{b}],[\mathrm{d}],[\mathrm{g}],[\mathrm{p}],[\mathrm{t}]$, and $[k]$. These six stops differ from each other either in place of articulation, in voicing, or in both place and voicing. One error analysis was concerned with double contrast pairs, i.e., those pairs which contrast in both voicing and place (e.g., [ba] and [ta]). On these trials, the percentage of blending responses, which consist of the pairing of the voicing value from one stimulus with the place value from the other stimulus (e.g., a response of [pa] or [da]). was much greater than would be expected by chance. On the basis of these results, it was argued that during processing the stimuli are analyzed into their component features and that the features from the two signals interact prior to being regrouped (sometimes erroneously) into phonetic units.

A second error analysis involved the conditions under which the stimuli presented to the two ears were correctly identified. It was found that the probability of double correct responses was a function of whether the stimuli shared a feature value, with shared pairs (e.g., [ba]-[da] which share voicing or [ba]-[pa] which share place) yielding a higher percentage of two correct responses than double contrast pairs. From the results on shared feature pairs, it was argued that the two signals converge prior to phonetic feature extraction, and that the feature value assigned to one stimulus is at least in part a function of whether the second stimulus also has that feature value.

Consider the adaptation effect and the model proposed to account for it in relation to these dichotic listening findings. If the site of adaptation is either prior to or coincident with the site of feature extraction and interaction, and if information regarding the relative strength of the detector's output is registered by higher processing centers, adaptation would be expected to result in a systematic alteration in the pattern of interaction of the stimuli in the two ears. For the purposes of explication, take the case where the four stimuli, [ba], [da], [pa], and [ta], are paired with each other to form the six dichotic pairs, [ba]-[da], [ba]-[pa], [ba]-[ta], [da]-[pa], [da]-[ta], and [pa]-[ta]. The feature dimension in question is voicing, and the listener's task is to report the identity of the consonant in a given ear. It would be expected, given the Studdert-Kennedy and Shankweiler findings, that prior to adaptation the voicing feature would be identified correctly a high percentage of the time on those trials on which both stimuli have the same voicing value. For the pairs which contrast in voicing, however, a certain percentage of errors on voicing identification would be expected, such that the stimulus in a given ear would be incorrectly assigned the voicing value of the opposing stimulus. For example, for the [ba]-[pa] pair, sometimes the [ba] would be identified as [pa] and sometimes the [pa] would be identified as [ba].

Now consider the effect of adaptation with a voiced adapting stimulus. According to the model, this would result in decreased output of the voiced detector channel for all voiced stimuli, including good exemplars of [ba] and [da]. This diminution of output of the voiced detector, if registered by higher processing centers, would result in the decreased effectiveness of the voiced stimulus when competing for processing with the voiceless stimulus. This reduction in effectiveness would be revealed in two ways. First, voiced stimuli, when paired with voiceless stimuli, would be incorrectly identified as voiceless more often (compared to preadaptation). That is, the unadapted voiceless value of the contrasting stimulus would cause relatively more interference after adaptation with the processing of the adapted voiced value of the target stimulus. Second, voiceless targets, when paired with voiced stimuli, would be identified correctly more often after than before adaptation, due to decreased interference from the adapted feature value of the opposing voiced stimulus. An analagous set of predictions can be made for the effects of adaptation with a voiceless stimulus. In this case, the adapted voiceless feature value would be identified correctly less often, and the unadapted voiced feature value would be identified correctly more often, after adaptation.

It is further predicted that the effect of adaptation would only be seen for those pairs which contrast in voicing, i.e., where one stimulus is voiced and the other is voiceless. Stimuli which share the voicing value would not be expected to show the effects of adaptation. Voiced adaptation, for example, would not affect identification of the voiceless pair ([pa]-[ta]) since neither stimulus has the adapted feature value. Furthermore, no change would occur for the voiced pair ([ba]-[da]) as well, since even though both stimuli have the adapted value, it is the same value, and thus there is no competition. In essence, this situation is analagous to the typical binaural adaptation procedure, which yields no change in identification of good exemplars of the adapted phonetic category. 
The existence of the adaptation effects described above would strongly suggest that (1) adaptation does affect a detector's sensitivity across a wide range of adequate stimulus values and not only at the values near the phonetic boundary, and that (2) information regarding the output level of the detector is registered and used by higher levels of processing. In order to test for these effects, two experiments were run in which a dichotic listening task was combined with a selective adaptation procedure. Experiment I investigated the effects of adaptation with the voicing feature, and Experiment II extended the paradigm to investigate the place of articulation dimension. The primary question of interest in both studies was the effect of adaptation on the interaction of the adapted feature of the stimuli in the two ears.

\section{EXPERIMENT I}

\section{Method}

Subjects. The subjects were eight volunteer Brown University students who were paid for their participation in the experiment. All were native speakers of English with no known speech or hearing disorder. Each of the subjects had had prior experience listening to synthetic speech.

Stimuli. The stimuli used for both adaptation and testing were the four syllables [ba], [da], [pa], and [ta]. They were generated on the Haskins Laboratories' parallel resonance synthesizer and formed a matched set, differing from each other only in place of articulation, voicing, or both place and voicing. The place of articulation distinction between [ba]-[pa] and [da]-[ta] was signaled by differences in the second and third formant transitions. and the voicing distinction between [ba]-[da] and [pa]-[ta] was signaled by differences in VOT. Each stimulus was $300 \mathrm{msec}$ in duration, with an initial transition of $45 \mathrm{msec}$ followed by a 255 -msec steady-state segment. The steady-state frequency values of the stimuli were identical: $769 \mathrm{~Hz}$ for $F 1,1,232 \mathrm{~Hz}$ for $F 2$, and $2,525 \mathrm{~Hz}$ for F3. For all four stimuli, F1 began at $537 \mathrm{~Hz}$. For [ba] and [pa], F2 began at $921 \mathrm{~Hz}$ and $\mathrm{F} 3$ began at $2,018 \mathrm{~Hz}$, and for [da] and [ta], the initial $\mathrm{F} 2$ and $F 3$ frequency values were 1.695 and $3.363 \mathrm{~Hz}$, respectively. During the transition segment, the frequencies changed linearly over time from the starting values to the steady-state values. The [ba] and [da] stimuli had a VOT value of $0 \mathrm{msec}$, whereas the [pa] and [ta] tokens had a $+50 \mathrm{msec}$ VOT value. The four syllables had identical overall amplitude and fundamental frequency contours.

Two adapting loops were prepared, one for each of the adaptation sessions. The loop for the voiced session contained a sequence of alternating [ba]s and [da]s, and the loop for the voiceless session contained a sequence of alternating [pa]s and [ta]s. In each case, the stimuli were recorded with an interstimulus interval of $350 \mathrm{msec}$.

It is possible to pair each of the four stimuli with each of the other three stimuli in six ways. For each of the six resulting pairs, there are two possible channel assignments, yielding 12 configurations. These 12 configurations formed the basis of the test tapes. Each tape contained six instances of each of the 12 configurations, for a total of 72 trials. The order of the pairs was randomized, with the restriction that each pair occur equally often in the first and second halves of the tape. Four such dichotic tapes were prepared using the Haskins PCM system (Cooper \& Mattingly, 1969). The stimuli on the two channels had simultaneous onsets and offsets, and a 3-sec interval between pairs was maintained throughout.

Apparatus. The basic instrumentation system for presenting the stimuli consisted of an Ampex AG-500 tape recorder, a Crown 820-144 tape recorder, and four matched sets of Koss Pro 600AA earphones. The output signals of Channels 1 and 2 of the Crown were shunted to the inputs of Channels 1 and 2, respectively, of the Ampex, whose outputs were sent concurrently to the four sets of earphones. With an appropriate switching apparatus, it was possible to shunt either the output of the Crown or the Ampex to the earphones, with either the output of the two channels going to different ears or the output of one channel passed to both ears. The adapting loops were played on the Ampex (Channel 1), while the test tapes were played on the Crown (Channels 1 and 2). With this system, it was possible to switch from adapting to test stimuli, and from binaural to dichotic presentation, within a few seconds. All stimuli were presented at $80 \mathrm{~dB} \mathrm{SPL}$, measured at the earphones with a General Radio sound-level meter.

Procedure. Two groups of four subjects each were run in two sessions. In one session, the subjects were adapted with voiced stimuli (voiced session), and in the other session, they were adapted with voiceless stimuli (voiceless session). The sessions were run on separate days, with one group receiving voiced adaptation on the first day and the other receiving voiced adaptation on the second day.

Each session consisted of three conditions: monotic identification, preadaptation dichotic identification, and postadaptation dichotic identification. The subject's task in all three conditions was identical. He was to monitor the stimuli in one ear, and to report which of the four syllables he heard by writing the appropriate letter on a prepared answer sheet. In the monotic condition, no stimulus was present in the unmonitored ear, while in both of the dichotic conditions, a contrasting stimulus was present on each trial.

Two tapes (144 items) were identified in each condition, with different tapes used in the preadaptation and postadaptation dichotic conditions. Each subject monitored the right ear for half of the trials and the left ear for the other half of the trials, with half of the subjects always monitoring the right ear on the first tape and the left ear on the second tape, and half monitoring in the reverse order. Tape channel-to-ear assignment was counterbalanced across the monitored ear by having subjects reverse the earphones after the first 36 trials. and then again after the next 72 trials.

For the monotic and preadaptation conditions, the subjects simply identified the stimuli, taking a short break after every 36 trials. The adaptation condition proceeded as follows. The subjects were first binaurally presented the adapting stimuli for $3 \mathrm{~min}(92$ stimulus presentations per minute), and then were presented six items for identification. They were then given another minute of adaptation. followed by six additional stimuli to identify. This sequence of $1 \mathrm{~min}$ of adaptation followed by six identification trials continued until 36 stimuli had been identified. This entire sequence of adaptation and identification (beginning with a 3-min adaptation period) was repeated three times, so that a total of 144 stimuli were identified in all.

\section{Results and Discussion}

Monotic identification was very good. Each of the four stimuli was identified correctly on over $98 \%$ of the trials by all subjects in both sessions.

The dichotic identification data were analyzed in a number of ways. The first analysis was concerned with overall correct performance. For a response to be scored as correct, both the voicing feature and the place of articulation feature had to be identified correctly. An analysis of variance, Session (voiced vs. voiceless adaptation) by Condition (pre- vs. postadaptation) by Ear (right vs. left), was performed on these scores. The analysis revealed two significant main effects, one due to ear, $F(1,7)=12.38, p<.01$, and one due to session, $F(1,7)=6.86, p<.05$. The ear effect was due to better performance, in each condition of each session, on stimuli monitored in the 
right ear. Across sessions, right-ear performance averaged $59 \%$ correct compared to $38 \%$ correct for the left ear. Thus the REA typically found for stop consonants was also present in this study, and was present both before and after adaptation. The session effect was due to a higher level of performance in the voiceless adaptation session, during both pre- and postadaptation. ${ }^{2}$

For the next analysis, the responses were rescored for correct identification of the voicing feature, irrespective of performance on the place feature. An analysis of variance on these scores (Session by Condition by Ear) also showed two significant effects, one due to ear, $F(1,7)=9.98, p<.025$, and one due to session, $F(1,7)=7.73, p<.05$. Thus scoring the responses either for performance on both the voicing and the place feature or for identification of the voicing feature alone resulted in the same pattern of results.

The lack of a condition effect, in both analyses, signified that adaptation did not result in a change in the level of performance. However, it is possible to maintain overall performance level while altering underlying patterns of behavior. One such pattern involves the extent to which a particular feature value is reported. It had been hypothesized that adaptation would result in fewer responses assigned to the adapted feature value. In order to test for this effect, the responses were scored simply for the presence of the adapted feature value. An analysis of variance, Adapted Feature Value (voiced vs. voiceless) by Condition (pre- vs. postadaptation) by Ear (right vs. left), indicated two significant effects, one due to condition, $F(1,7)=16.48, p<.01$, and one due to the Condition by Adapted Feature Value interaction, $F(1,7)=9.63, p<.025$. The condition effect was the result of significantly fewer responses with the adapted feature value following adaptation. Thus, in the voiced adaptation session, there were fewer voiced responses following adaptation, and in the voiceless session, there were fewer voiceless responses following adaptation. The interaction was due to a larger prevs. postadaptation difference for adaptation with the voiceless feature value.

In order to investigate further the nature of this effect, performance on specific pairs was considered. The six possible dichotic pairs can be divided into two types, shared pairs and contrast pairs. In terms of whether the target stimulus has the same voicing value as the adapting stimuli, there are really two kinds of shared pairs and two kinds of contrast pairs. With respect to the shared pairs, in one type both the target and the nontarget stimulus have the same feature value as the adapting stimuli. For the second kind of shared pair, both the target and the nontarget stimulus have the unadapted feature value. In one type of contrast pair, the target stimulus has the adapted feature value and the opposing stimulus has the unadapted feature value. With the second type of contrast pair, the converse is true: the target stimulus has the unadapted feature value and the nontarget stimulus has the adapted feature value. Of interest is the change in performance on each of these kinds of pairs after adaptation.

In order to test for the effect of adaptation on these kinds of pairs, the following analysis was carried out. First, each response was scored for accuracy with respect to the voicing feature alone. The number of correct responses for each of the four types of pairs was tallied separately according to the ear monitored. These data, averaged across subjects and expressed in terms of percentages, are presented in Table 1. Next, for each type of pair, a mean difference score was calculated by subtracting the mean number of correct responses prior to adaptation from the mean number of correct responses following adaptation. Thus, a positive difference score indicated improved performance and a negative difference score indicated a decrease in performance level. For example, Table 1 shows that during the voiceless adaptation session, when targeting for the unadapted feature value in the left ear, preadaptation performance averaged $31 \%$ correct. This corresponds to a mean of 1.88 correct responses. During postadaptation, performance averaged $70 \%$ correct, which corresponds to a mean of 4.22 correct voicing identifications. The difference score for this cell would thus be +2.34 .

First the results for the shared pairs will be considered. Inspection of the data indicated that in both sessions subjects performed almost perfectly both before and after adaptation. Individual analyses on the difference scores showed no reliable change in performance following adaptation for either the adapted or unadapted feature value $(p>.10$ in all cases). That is, the subjects nearly always correctly identified the voicing value of the target stimulus when the nontarget stimulus had the same voicing value.

Next consider the contrast pairs. Unlike the shared pairs, preadaptation performance was considerably below $100 \%$ correct, and adaptation did result in systematic shifts in response patterns. The difference scores for the contrast pairs were entered into an

Table 1

Mean Percentage of Correct Voicing Responses During Preand Postadaptation, as a Function of Voiced vs. Voiceless Adaptation, Adapted vs. Unadapted Target Value, and Right vs. Left Ear, for Shared Pairs and Contrast Pairs

\begin{tabular}{|c|c|c|c|c|c|c|c|c|c|}
\hline \multirow{3}{*}{$\begin{array}{c}\text { Pair } \\
\text { Type }\end{array}$} & \multirow{3}{*}{$\begin{array}{c}\text { Target } \\
\text { Value }\end{array}$} & \multicolumn{4}{|c|}{ Voiced Adaptation } & \multicolumn{4}{|c|}{$\begin{array}{l}\text { Voiceless } \\
\text { Adaptation }\end{array}$} \\
\hline & & \multicolumn{2}{|c|}{ Pre } & \multicolumn{2}{|c|}{ Post } & \multicolumn{2}{|c|}{ Pre } & \multicolumn{2}{|c|}{ Post } \\
\hline & & $\mathbf{R}$ & $\mathbf{L}$ & $\mathbf{R}$ & $\mathbf{L}$ & $\mathbf{R}$ & $\mathbf{L}$ & $\mathbf{R}$ & $\mathbf{L}$ \\
\hline Shared & $\begin{array}{l}\text { Adapted } \\
\text { Unadapted }\end{array}$ & $\begin{array}{l}100 \\
100\end{array}$ & $\begin{array}{l}100 \\
100\end{array}$ & $\begin{array}{l}97 \\
99\end{array}$ & $\begin{array}{l}98 \\
99\end{array}$ & $\begin{array}{l}99 \\
99\end{array}$ & $\begin{array}{r}98 \\
100\end{array}$ & $\begin{array}{l}100 \\
100\end{array}$ & $\begin{array}{l}100 \\
100\end{array}$ \\
\hline Contrast & $\begin{array}{l}\text { Adapted } \\
\text { Unadapted }\end{array}$ & $\begin{array}{l}39 \\
87\end{array}$ & $\begin{array}{l}26 \\
58\end{array}$ & $\begin{array}{l}30 \\
94\end{array}$ & $\begin{array}{l}17 \\
82\end{array}$ & $\begin{array}{l}87 \\
62\end{array}$ & $\begin{array}{l}56 \\
31\end{array}$ & $\begin{array}{l}44 \\
84\end{array}$ & $\begin{array}{l}28 \\
70\end{array}$ \\
\hline
\end{tabular}


analysis of variance. Feature Value Adapted (voiced vs. voiceless) by Target (adapted vs. unadapted feature value) by Ear (right vs. left). There was a significant effect due to target, $F(1,7)=16.78$, $\mathrm{p}<.01$, and an effect due to the Target by Feature Value Adapted interaction, $F(1,7)=9.83, \mathrm{p}<.025$. The mean difference scores for the contrast pairs, as a function of feature value adapted and target, are displayed in Figure 2. As can be seen in this figure, the target effect was due to decreased performance after adaptation when targeting for the adapted feature value (negative difference score) as well as improved performance when targeting for the unadapted feature value (positive difference score). Separate $t$ tests on these difference scores indicated that each was significantly different from zero ( $p<.025$ in all cases). That is, in both the voiced and voiceless adaptation sessions, adaptation resulted in a significant decrease in performance level when targeting for the adapted feature value and a significant increase in performance level when targeting for the unadapted feature value.

The interaction was the result of greater improvement for the unadapted value and a greater decrease in performance for the adapted value in the voiceless adaptation session. An asymmetry in the magnitude of the adaptation effect, ' with voiceless adaptation resulting in a greater effect, has also been

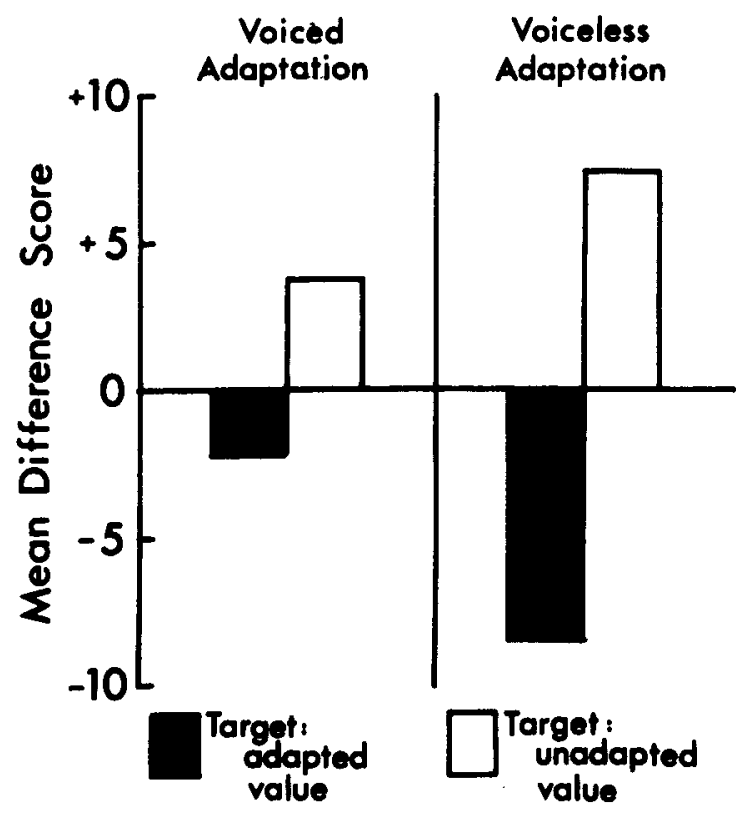

Figure 2. Mean difference in the number of correct volchng Judgments as a function of feature value adapted (voleed ve. volceless) and target feature value (adapted vo. unadapted). Difference scores were calculated by subtracting the number of correct responses on the volcing feature before adaptation from the number of correct respomes on the volcing feature after adaptation. Positive difference ccores indicate improved performance, and negative dlfference scores indicate depremed performance. found by investigators using the traditional binaural adaptation procedure. Eimas and Corbit (1973) found that adapting with a voiced stimulus resulted in less adaptation than adapting with a voiceless stimulus. In a further investigation of the effect, it was found that simultaneous adaptation using alternating voiced and voiceless adapting stimuli resulted in a net increase in the number of voiced responses, that is, a net effect due to the voiceless adapting stimulus (Eimas et al., 1973). As noted by Eimas et al., the direction of the asymmetry is consistent with certain data on speech production, which also indicate a primacy for the voiced feature value.

The results of this experiment clearly indicate that adaptation does affect the processing of nonboundary exemplars of a phonetic category. When a good exemplar that contains the adapted feature value is paired dichotically with an exemplar containing the unadapted value, the adapted value loses effectiveness. This occurs in two ways. First, the adapted feature value in the nontarget ear produces less interference during the processing of the unadapted value in the target ear. Second, the unadapted feature value in the nontarget ear interferes more with the processing of the adapted value in the target ear. Thus, during processing, there is an interaction between the two stimuli, and adaptation alters the relative potency of a given feature value in competing with the opposing feature value. Since these findings have theoretical implications for a detector model of feature extraction (as will be discussed below), it was deemed important to replicate the findings on a second linguistic dimension. Thus, a second experiment, investigating the effects of adaptation along the place of articulation dimension, was conducted using the same experimental paradigm.

\section{EXPERIMENT II}

\section{Method}

Subjects. The subjects from Experiment 1 also participated in this experiment.

Stimull. The four syllables used in Experiment $I$ also served as stimuli in this experiment. Testing was conducted with the tapes prepared for the first experiment. Two new loops were made for adaptation, one containing a sequence of alternating [ba]s and Ipa]s (labial adapting loop) and one containing a sequence of alternating [da]s and [ta]s (alveolar adapting loop). Again, the stimuli were recorded with an interstimulus interval of $350 \mathrm{msec}$.

Apparatus. The identical apparatus was used in this experiment as had been used in the first study.

Procedure. The procedure was identical to that of Experiment I except that in one session the subjects were adapted with labial stimuli and in the other session they were adapted with alveolar stimuli.

\section{Results and Discussion}

Monotic identification, as expected, was very good. As in Experiment $I$, each of the four stimuli was correctly identified on over $98 \%$ of the trials by all subjects in both sessions. 
The dichotic identification data were analyzed in an analogous manner to the data in the voicing experiment. The first analysis concerned overall performance with responses scored according to both place and voicing identification. An analysis of variance. Session (labial vs. alveolar) by Condition (pre- vs. postadaptation) by Ear (right vs. left), revealed two significant effects. First, there was an ear effect, $F(1,7)=9.07, p<.025$, which was due to the superiority of the right ear. Across subjects and sessions. right-ear performance averaged $60 \%$ correct compared to an average of $37 \%$ correct for the left ear. There was also a reliable Session by Condition by Ear interaction, $F(1,7)=9.78, p<.025$, which was due to a decrease in the relative ear advantage in the postadaptation condition of the alveolar adaptation session. Similar resuls were obtained when the responses were scored solely on the basis of place identification. Again, there was an ear effect, $F(1,7)$ $=6.48, p<.05$, and an effect due to the Session by Condition by Ear interaction, $F(1,7)=10.55$. $p<.025$.

As in the voicing experiment, there was no effect due to condition in either of the analyses of overall performance. In order to determine whether there were changes in underlying interactions of the place feature in the two ears, further analyses were conducted. First, the responses were scored simply for the presence of the adapted feature value. An analysis of variance. Adapted Feature Value by Condition by Ear showed an effect due to condition, $F(1,7)=$ 81.57, $p<.001$. After adaptation, there were significantly fewer responses with the adapted feature value. That is, there were fewer labial responses after labial adaptation and fewer alveolar responses after alveolar adaptation. There was also a significant Condition by Adapted Feature Value by Ear interaction $[F(1.7)=15.56, \mathrm{p}<.01] .^{3}$

An analysis of shared and contrast pairs, similar to that performed in the first experiment, was also done on these data. In this experiment, shared pairs were those that shared the place of articulation feature, whereas contrast pairs were those which differed in place of articulation. The results for the shared and contrast pairs, in terms of mean percent correct, are presented in Table 2 . The mean difference scores for

\section{Table 2}

Mean Percentage of Correct Place Responses During Pre and Postadaptation, as a Function of Labial vs. Alveolar Adaptation, Adapted vs. Unadapted Target Value, and Right vs. Left Ear, for Shared Pairs and Contrast Pairs

\begin{tabular}{|c|c|c|c|c|c|c|c|c|c|}
\hline \multirow{3}{*}{$\begin{array}{l}\text { Pair } \\
\text { Type }\end{array}$} & \multirow{3}{*}{$\begin{array}{l}\text { Target } \\
\text { Value }\end{array}$} & \multicolumn{4}{|c|}{$\begin{array}{c}\text { Labial } \\
\text { Adaptation }\end{array}$} & \multicolumn{4}{|c|}{$\begin{array}{c}\text { Alveolar } \\
\text { Adaptation }\end{array}$} \\
\hline & & \multicolumn{2}{|c|}{ Pre } & \multicolumn{2}{|c|}{ Post } & \multicolumn{2}{|c|}{ Pre } & \multicolumn{2}{|c|}{ Post } \\
\hline & & $\mathbf{R}$ & L & $\mathbf{R}$ & L & $\mathbf{R}$ & L & $\mathbf{R}$ & $\mathbf{L}$ \\
\hline Shared & $\begin{array}{l}\text { Adapted } \\
\text { Unadapted }\end{array}$ & $\begin{array}{r}100 \\
99\end{array}$ & $\begin{array}{l}99 \\
98\end{array}$ & $\begin{array}{r}97 \\
100\end{array}$ & $\begin{array}{r}100 \\
99\end{array}$ & $\begin{array}{l}100 \\
100\end{array}$ & $\begin{array}{r}99 \\
100\end{array}$ & $\begin{array}{l}97 \\
99\end{array}$ & $\begin{array}{r}96 \\
100\end{array}$ \\
\hline Contrast & $\begin{array}{l}\text { Adapted } \\
\text { Unadapted }\end{array}$ & $\begin{array}{l}68 \\
62\end{array}$ & $\begin{array}{l}48 \\
39\end{array}$ & $\begin{array}{l}34 \\
94\end{array}$ & $\begin{array}{r}8 \\
76\end{array}$ & $\begin{array}{l}60 \\
71\end{array}$ & $\begin{array}{l}35 \\
46\end{array}$ & $\begin{array}{l}22 \\
86\end{array}$ & $\begin{array}{l}24 \\
79\end{array}$ \\
\hline
\end{tabular}

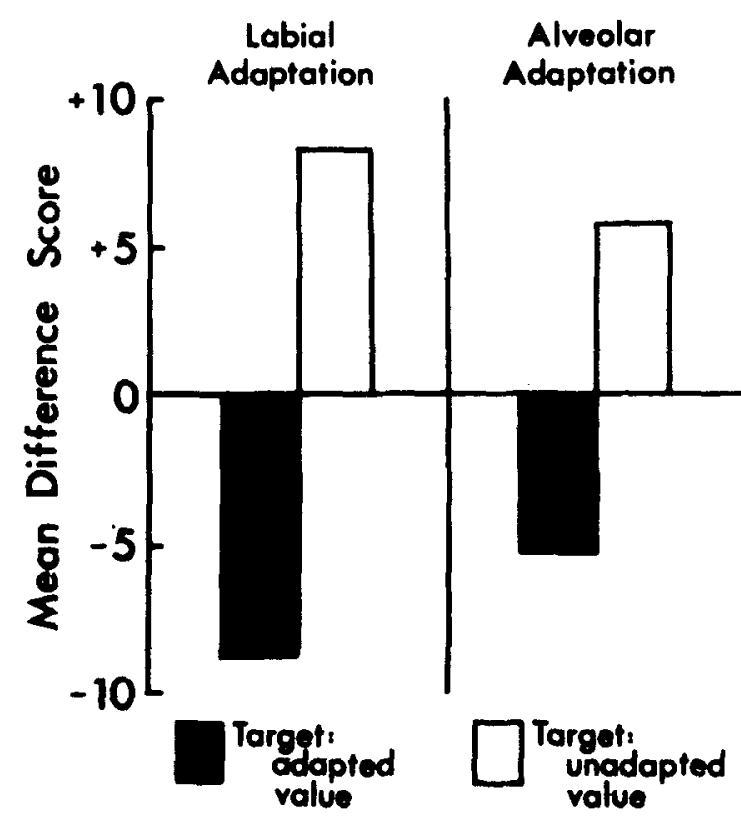

Figure 3. Mean difference in the number of correct place Judgmentw as a function of feature value adapted (labial $v$. alveolar) and turget feature value (adapted re. unadapted). Difference scores were calculated by mbtracthe the number of correct reaponses on the place fenture before adaptation from the number of correct responsen on the place feature afier aduptation. Posittre difference scores indicate improved performance, and negative difference scores Indkate depreweed performance.

the contrast pairs are graphically displayed in Figure 3.

The results for the shared pairs were similar to those that had been obtained in the voicing study. Performance, both before and after adaptation, was nearly perfect. Analyses of the mean difference scores indicated no effect of adaptation $(p>.10$ in all cases).

Performance on the contrast pairs did change as a function of adaptation. The difference scores were run in a Feature Value Adapted by Target by Ear analysis of variance, which revealed two reliable effects. The target effect, $F(1.7)=66.78, p<.001$, was due to a decrement in performance following adaptation (i.e.. negative difference scores) for target stimuli containing the adapted feature value, and improved performance following adaptation (hence positive difference scores) for target stimuli containing the unadapted feature value. Individual $t$ tests indicated that the mean difference scores for each of the conditions displayed in Figure 3 were significantly different from zero ( $p<.025$ in each case). The lack of a Target by Condition interaction signified that for the place feature, unlike for the voicing feature, the effect was symmetric with respect to the two feature values. labial and alveolar. That is, the difference between adapting stimuli that is discernible in Figure 3 was not reliable. There was also a significant Feature Value Adapted by Ear interaction. $F(1,7)=$ 7.55. $\mathrm{p}<.05$. Performance for the two ears did not differ reliably in the labial session. $t(7)=.16$. 
$p>.10$, but did differ in the alveolar session, $t(7)=$ $2.62, \mathrm{p}<.025$. In the alveolar session, the left ear showed a net gain in performance; that is, there was more improvement in targeting for the unadapted labial value than decrement in targeting for the adapted alveolar value. The right ear, however, showed a net loss, with relatively more decrement in targeting for the adapted alveolar value than improvement in targeting for the unadapted labial value. The reason for this is not clear.

In summary, the general pattern of results obtained with adaptation of the voicing feature was replicated on the place of articulation dimension. Pairs which shared either the adapted or the unadapted feature value showed no change due to adaptation. Performance on contrast pairs was systematically altered, indicating a change in the relative effectiveness of the adapted feature value during processing.

\section{GENERAL DISCUSSION}

The major findings for the two experiments were identical. For both the voicing feature and the place of articulation feature, the following set of results was obtained. First, after adaptation, when two nonboundary stimuli shared the adapted feature value and were presented dichotically, they were very nearly perfectly identified. There were few intrusions of the unadapted feature value, just as in the preadaptation condition. Second, when two nonboundary stimuli were presented dichotically, one having the unadapted feature value and one having the adapted feature value, postadaptation performance systematically differed from preadaptation performance. Prior to adaptation, there were errors on feature identification such that the target stimulus was sometimes erroneously assigned the feature value of the stimulus in the nontarget ear. Adaptation altered this error pattern in two ways. First, when the target stimulus had the adapted feature value, there were more errors following adaptation. That is, the unadapted value in the nontarget ear caused more interference (i.e., intruded more often) during the processing of the adapted target stimulus. In addition, when the target stimulus contained the unadapted feature value, it was identified correctly more often compared to preadaptation performance, being influenced less by the adapted feature value in the nontarget ear.

As indicated in the introduction, there is evidence that, at least for the typical binaural adaptation procedure, the shifts in performance following adaptation are not simply due to alterations in response criteria, but occur during some stage of perceptual processing (see Sawusch \& Pisoni, Note 1; Sawusch et al., Note 2). Provided that this is also true for the present adaptation procedure, the following set of arguments can be made.
(1) The fact that there was no effect of adaptation on the identification of two stimuli that shared the adapted feature value indicates that the output strength of the detector, although adapted, was still stronger than that of the unadapted detector at the point along the continuum at which the stimulus value was located.

(2) That adaptation significantly altered the identification of nonboundary stimuli indicates that the sensitivity of the detector was affected for a range of stimulus values. Presumably, straightforward identification procedures typically do not show adaptation effects for good exemplars of a category for the same reason that shared pairs, in the present studies, were not misidentified; namely, that the detector, although adapted, was still more strongly excited than the unadapted detector.

(3) Contrary to the original assumption of Eimas and Corbit (1973), the relative output strength of the detector is registered by higher centers of processing. If this were not the case, there would be no reason to predict that adaptation would affect the processing of nonboundary stimuli. In the dichotic situation, each stimulus is assigned a feature value. The results from the shared pairs indicate that which feature value is assigned does not change as a function of adaptation. Since the relative potency of a feature value does change as a result of adaptation (as indicated by performance on contrast pairs), additional information must be registered by higher centers of processing, namely, information regarding the relative strength of the output from the maximally excited detector. Alteration in error patterns occurs because adaptation yields a weakened output signal, which is registered by higher processing centers, and which results in less competition when interacting with the unadapted value in the other ear.

(4) The site of adaptation cannot be subsequent to the interaction of the stimulus information from the two ears. Interaction patterns change because the relative levels of the graded outputs change. Thus, the interaction of the two stimuli must occur during a stage of processing at which the information concerning the relative output levels of the detectors, as a function of adaptation, is still available."

The results of these experiments provide behavioral evidence for the existence of detector mechanisms that extract information relevant for the assignment of phonetic feature values, and support certain specific assumptions concerning the operation of the detector systems. There are a number of important issues that remain to be explored, including the level(s) at which the detectors are operating, the output function (or the slope) of the detector response before and after adaptation, and the relative amount of adaptation as a function of location along the feature dimension. Further development of the dichotic adaptation paradigm may provide an empirical means for investigation of these issues. 


\section{REFERENCE NOTES}

1. Sawusch, J. R., \& Pisoni. D. B. Category boundaries in speech and nonspeech sounds. Paper presented at the 86th meeting of the Acoustical Society of America, 1973, Los Angeles.

2. Sawusch, J. R., Pisoni, D. B., \& Cutting, J. E. Category boundaries for linguistic and nonlinguistic dimensions of the same stimuli. Paper presented at the 87th meeting of the Acoustical Society of America, 1974, New York.

\section{REFERENCES}

AbBs, J. H., \& Sussman, H. M. Neurophysiological feature detectors and speech perception: A discussion of theoretical implications. Journal of Speech and Hearing Research, 1971. 14. 23-36.

Ades, A. E. Bilateral component in speech perception? Journal of the Acoustical Society of America, 1974, 56, 610-617. (a)

ADES, A. E. How phonetic is selective adaptation? Experiments on syllable position and vowel environment. Perception \& Psychophysics, 1974, 16, 61-66. (b)

ANDERSON, F. Some implications for the operation of feature detectors in speech perception: Use of identification on response times as a converging operation. Unpublished $\mathrm{PhD}$ thesis, Brown University, 1975.

Cooper, F. S., \& Mattingly, I. G. Computer-controlled PCM system for investigation of dichotic speech perception. Journal of the Acoustical Society of America, 1969, 46, 115 (A).

COOPER, W. E. Adaptation of phonetic feature analyzers for place of articulation. Journal of the Acoustical Society of America, 1974, 56, 617-628. (a)

Cooper, W. E. Selective adaptation for acoustic cues of voicing in initial stops. Journal of Phonetics, 1974, 2, 303-313. (b)

Cooper. W. E., \& Blumstein, S. E. A labial feature analyzer in speech perception. Perception \& Psychophysics, 1974, 15, 591-600.

DIEHL. R. H. The effect of selective adaptation on the identification of speech sounds. Perception \& Psychophysics, 1975, 17, 48-52.

Eimas, P. D., Cooper, W. E., \& Corbit, J. D. Some properties of linguistic feature detectors. Perception \& Psychophysics, 1973, 13, 247-252.

Eimas. P. D.. \& Corbit, J. D. Selective adaptation of linguistic feature detectors. Cognitive Psychology, 1973, 4, 99-109.

Liberman, A. M.. Cooper, F, S., Shankweiler, D. P., \& Studdert-Kennedy, M. Perception of the speech code. Psychological Review, 1967, 74, 431-461.

LISKER, L., \& ABRAmson, A. S. A cross-language study of voicing in initial stops: Acoustical measurements. Word, 1964 , 20. $384-422$.

Pisoni. D. B.. \& TASH, J. Reaction time to comparisons within and across phonetic categories. Perception \& Psychophysics. 1974, 15. 285-291.
Sekuler, R. Spatial vision. Annual Review of Psychology, 1974, 25, 195-232.

Studdert-Kennedy, M. The perception of speech. In T. A. Sebeok (Ed.). Current trends in linguistics. Vol. XII. The Hague: Mouton, 1974

Studdert-Kennedy, M., \& Shankweiler, D. Hemispheric specialization for speech perception. Journal of the Acoustical Society of America, 1970, 48, 579-594.

TartTeR, V., \& Ermas, P. The role of auditory feature detectors in the perception of speech. Perception \& Psychophysics, in press.

WARD, W. D, Adaptation and fatigue. In J. Jerger (Ed.), Modern developments in audiology. New York: Academic Press, 1973

\section{NOTES}

1. The distinction between auditory and phonetic feature extraction is certainly critical, as is the specification of the kinds of detector systems operating at each level of analysis. Whereas the design of the current studies precluded determining whether the effects of adaptation interacted with auditory or phonetic feature processing (e.g., identical adapting and test stimuli were used), experiments are currently being designed that will investigate the characteristics of the detectors at each level of analysis.

2 . This effect was probably due to sampling error. Performance differed in the preadaptation as well as in the postadaptation conditions of the two sessions, even though the two preadaptation conditions were identical in terms of experimental procedure.

3. The differences were quite small, and only reached significance because of minimal variability. The direction of the differences was as follows. In the labial adaptation session, the left ear yielded more labial responses during preadaptation and the right ear gave more labial responses after adaptation. In the alveolar session, the right ear provided more alveolar responses before adaptation whereas the left year yielded a greater number of alveolar responses following adaptation.

4. Ades (1974a) also used a selective adaptation technique to investigate the relative location of property detectors for speech. He found evidence both for adaptation effects at an ear-dependent level and for effects at a more central level, i.e., after the point of binaural fusion. The data from the present studies indicate only that at least some component of adaptation occurs prior to the interaction of information from the two ears.

(Received for publication June 20, 1975; revision received August 15, 1975.) 